Pacific Journal of Mathematics

DECOMPOSITION THEOREMS FOR FREDHOLM OPERATORS 


\title{
DECOMPOSITION THEOREMS FOR FREDHOLM OPERATORS
}

\author{
T. W. GAMELIN
}

This paper is devoted to proving and discussing several consequences of the following decomposition theorem:

Let $A$ and $B$ be closed densely-defined linear operators from the Banach space $X$ to the Banach space $Y$ such that $D(B) \supseteqq D(A), D\left(B^{*}\right) \supseteq D\left(A^{*}\right)$, the range $R(A)$ of $A$ is closed, and the dimension of the null-space $N(A)$ of $A$ is finite. Then $X$ and $Y$ can be decomposed into direct sums $X=X_{0} \oplus X_{1}$, $Y=Y_{0} \oplus Y_{1}$, where $X_{1}$ and $Y_{1}$ are finite dimensional, $X_{1} \subseteq D(A)$, $X_{0} \cap D(A)$ is dense in $X$, and $\left(X_{0}, Y_{0}\right)$ and $\left(X_{1}, Y_{1}\right)$ are invariant pairs of subspaces for both $A$ and $B$. Let $A_{i}$ and $B_{i}$ be the restrictions of $A$ and $B$ respectively to $X_{i}$. For all integers $k,\left(B_{0} A_{0}^{-1}\right)^{k}(0) \subseteq R\left(A_{0}\right)$, and $\operatorname{dim}\left(B_{0} A_{0}^{-1}\right)^{k}(0)=k \operatorname{dim}\left(B_{0} A_{0}^{-1}\right)(0)=k \operatorname{dim} N\left(A_{0}\right)$.

Also, the action of $A_{1}$ and $B_{1}$ from $X_{1}$ to $Y_{1}$ can be given a certain canonical description.

The object of this paper is to study the operator equation $A x-\lambda B x=y$, where $A$ and $B$ are (unbounded) linear operators from a Banach space $X$ to a Banach space $Y$. In $\S 1$, an integer $\mu(A: B)$ is defined, which expresses a certain interrelationship between the null space of $A$ and the null space of $B$. In $\S 1$ and 2 , decomposition theorems are proved which refine theorem 4 of [2]. The theorems allow us to split off certain finite dimensional invariant pairs of subspaces of $X$ and $Y$ so that $A$ and $B$ are well-behaved with respect to $\mu(A: B)$ on the remainder.

In $\S 4$, the stability of these decompositions under perturbation of $A$ by $\lambda B$ is investigated. In $\S 5$, relations between the dimensions of certain subspaces of $X$ and $Y$ are given, and a formula for the Fredholm index of $A-\lambda B$ is obtained. These extend results of Kaniel and Schechter [1], who consider the case $X=Y$ and $B$ the indentity operator.

It should be noted that the results of Kaniel and Schechter referred to here follow from theorems 3 and 4 of [2]. The results of this paper properly refine Kato's results only when the null space of $B$ is not $\{0\}$.

1. We will be considering linear operators $T$ defined on a dense linear subset $D(A)$ of a Banach space $X$, and with values in a Banach space $Y . N(T)$ and $R(T)$ will denote the null space and range of $T$ respectively, while $\alpha(T)$ is the dimension of $N(T)$, and $\beta(T)$ is the

Received February 10, 1964. 
codimension of $\overline{R(T)}$ in $Y . \quad T$ is a Fredholm operator if $T$ is closed, $R(T)$ is closed, and both $\alpha(T)$ and $\beta(T)$ are finite. The index of a Fredholm operator is the integer.

$$
\kappa(T)=\alpha(T)-\beta(T) .
$$

Let $P$ be a subspace of $X, Q$ a subspace of $Y . \quad(P, Q)$ is an invariant pair of subspaces for $T$ if $T(P \cap D(T)) \subseteq Q$.

Standing assumptions: In the remainder of the paper, $A$ and $B$ are closed linear operators from $X$ to $Y, D(A)$ is dense in $X, D(B) \supseteqq D(A)$, and $D\left(B^{*}\right) \supseteqq D\left(A^{*}\right) ; A$ is semi-Fredholm, in the sense that $R(A)$ is closed and $\alpha(A)<\infty$.

The assumption $D\left(B^{*}\right) \supseteqq D\left(A^{*}\right)$ seems necessary for the proof of the decomposition theorems. It is often met when $A$ and $B$ are differential operators on some domain in Euclidean space, and the order of $B$ is less than the order of $A$. It is always met when $B$ is bounded.

The linear manifolds $N_{k}=N_{k}(A: B)$ and $M_{k}=M_{k}(A: B)$ are defined by induction as follows:

$$
\begin{aligned}
& N_{1}=N(A) \\
& N_{k}=A^{-1}\left(B N_{k-1}\right), \quad k>1 \\
& M_{k}=B N_{k} .
\end{aligned}
$$

$N_{k}$ and $M_{k}$ are increasing sequences of linear manifolds in $X$ and $Y$ respectively.

The smallest integer $n$ such that $N_{n}$ is not a subset of $B^{-1} R(A)$ will be denoted by $\nu(A: B)$. If $N_{n}$ is a subset of $B^{-1} R(A)$ for all $n$, then we define $\nu(A: B)=\infty$. (cf. [2])

The dimension of $N_{k}$ will be denoted by $\pi_{k}=\pi_{k}(A: B)$, and the dimension of $M_{k}$ by $\rho_{k}=\rho_{k}(A: B)$. Then $\pi_{1}=\alpha(A)$, and, in general, $\pi_{k} \leqq k \alpha(A) . \quad \mu(A: B)$ will denote the first integer $n$ such that $\pi_{n}<n \alpha(A)$. It $\pi_{n}=n \alpha(A)$ for all intergers $n$, then we define $\mu(A: B)=\infty$.

In general, $\mu(A: B) \geqq \nu(A: B)+1$. This inequality is trivial if $\nu_{\lrcorner}=\infty$. If $\nu<\infty$, then $M_{\nu-1} \subseteq R(A)$, while $M_{\nu} \nsubseteq R(A)$. Consequently, $\pi_{\nu+1}<\pi_{\nu}+\alpha(A) \leqq(\nu+1) \alpha(A)$, and so $\mu(A: B) \leqq \nu+1$.

We define $\sigma_{k}(A: B)=\pi_{k}-\pi_{k-1}$. Then $\sigma_{k}$ is the dimension of the quotient space $N_{k} / N_{k-1}$. $\quad\left\{\sigma_{k}\right\}$ is a decreasing sequence of nonnegative integers, and so the limit

$$
\sigma(A: B)=\lim _{k \rightarrow \infty} \sigma_{k}(A: B) \quad \text { exists. }
$$

If $\mu(A: B)=\infty$, then $\sigma(A: B)=\alpha(A)$.

2. THEOREM 1. Assume, in addition to the standing assumptions on $A$ and $B$, that $\nu(A: B)=\infty$. Then $X$ and $Y$ can be decomposed 
into direct sums

$$
\begin{aligned}
& X=X_{0} \oplus X_{1} \\
& Y=Y_{0} \oplus Y_{1},
\end{aligned}
$$

where $X_{1}$ and $Y_{1}$ are finite dimensional, $X_{1} \subseteq D(A), X_{0} \cap D(A)$ is dense in $X_{0}$, and $\left(X_{0}, Y_{0}\right)$ and $\left(X_{1}, Y_{1}\right)$ are invariant pairs for both $A$ and $B$. If $A_{i}$ and $B_{i}$ are the restrictions of $A$ and $B$ respectively to $X_{i}$, then $\mu\left(A_{0}, B_{0}\right)=\infty$, while $A_{1}$ and $B_{1}$ map $X_{1}$ onto $Y_{1}$.

Furthermore, $X_{1}$ and $Y_{1}$ can be decomposed as direct sums

$$
\begin{aligned}
& X_{1}=P_{1} \oplus \cdots \oplus P_{p} \\
& Y_{1}=Q_{1} \oplus \cdots \oplus Q_{p},
\end{aligned}
$$

where $A_{1}$ and $B_{1}$ map $P_{j}$ onto $Q_{j}$. Bases $\left\{x_{j}^{i}: 1<i \leqq \eta(j)\right\}$ and $\left\{y_{j}^{i}: 1 \leqq i \leqq \eta(j)-1\right\}$ can be chosen for $P_{j}$ and $Q_{j}$ respectively so that

$$
\begin{aligned}
& A x_{j}^{i+1}=B x_{j}^{i}=y_{j}^{i}, \quad 1 \leqq i \leqq \eta(j)-1 \\
& A x_{j}^{1}=0=B x_{j}^{\eta(j)} .
\end{aligned}
$$

Although the decomposition is not, in general, unique, the integers $p$ and $\eta(j), 1 \leqq j \leqq m$, are uniquely determined by $A$ and $B$. In fact,

$$
p=\alpha(A)-\sigma(A: B) .
$$

Proof. Let $n=\alpha(A)$, and suppose that $\left\{z_{1}^{1}, \cdots z_{n}^{1}\right\}$ is a basis for $N(A)$. Since $\nu(A: B)=\infty, z_{j}^{i}$ can be chosen by induction so that $A z_{j}^{i}=B z_{j}^{i-1} . \quad\left\{z_{j}^{i}: 1 \leqq j \leqq n, 1 \leqq i \leqq m\right\}$ is a spanning set for $N_{m}$, while $\left\{B z_{j}^{i}: 1 \leqq j \leqq n, 1 \leqq i \leqq m\right\}$ is a spanning set for $M_{m}$. Also, $\left\{z_{i}^{m}: 1 \leqq i \leqq n\right\}$ span $N_{m}$ modulo $N_{m-1}$.

Recall that $\sigma_{m}=\sigma(m)=\operatorname{dim}\left(N_{m} / N_{m-1}\right)$. By induction, the order of the $z_{j}^{i}$ can be chosen so that $\left\{z_{n-\sigma(m)+1}^{m}, \cdots, z_{n}^{m}\right\}$ span $N_{m}$ modulo $N_{m-1}$. Then

$$
G_{m}=\left\{z_{j}^{i}: n-\sigma(i)+1 \leqq j \leqq n, 1 \leqq i \leqq m\right\}
$$

is a basis for $N_{m}$.

Let $\eta(j)$ be the greatest integer $k$ such that $z_{j}^{k} \in G_{k}$. If $z_{j}^{k} \in G_{k}$ for all $k$, let $\eta(j)=\infty$. Then $1 \leqq \eta(1) \leqq \eta(2) \leqq \cdots \leqq \eta(n)$. Let $p$ be the greatest integer $k$ such that $\eta(k)<\infty$. By definition of $\sigma$, it is clear that

$$
p=\alpha(A)-\sigma \text {. }
$$

Suppose $1 \leqq j \leqq p . \quad z_{j}^{\eta(j)+1}$ is linearly dependent on the set $G_{\eta(j)+1}$, and so we can write 


$$
z_{j}^{\eta(j)+1}=\sum \alpha_{i k} z_{k}^{i},
$$

where the sum is taken over all pairs of integers $(i, k)$, with the understanding that $z_{k}^{i}=0$ if $i \leqq 0$ and $\alpha_{i k}=0$ if $z_{k}^{i} \notin G_{\eta(j)+1}$. For $-1 \leqq q \leqq \eta(j)$ define

$$
x_{j}^{\eta(j)-q}=z_{j}^{\eta(j)-q}-\sum \alpha_{i k} z_{k}^{i-q-1} .
$$

For $0 \leqq q \leqq \eta(j)$,

$$
\begin{aligned}
B x_{j}^{\eta(j)-q} & =B z_{j}^{\eta(j)-q}-\sum \alpha_{i k} B z_{k}^{i-q-1} \\
& =A z_{j}^{\eta(j)-q+1}-\sum \alpha_{i k} A z_{k}^{i-q} \\
& =A x_{j}^{\eta(j)-q+1}
\end{aligned}
$$

In particular, $B x_{j}^{\eta(j)}=0$.

Since the sum for $x_{j}^{\eta(j)-q}$ involves $z_{j}^{\eta(j)-q}$ only in the first term, the $z_{j(j)-q}^{\eta}$ may be replaced by the $x_{j}^{\eta(j)-q}, 0 \leqq q \leqq \eta(j)$, to obtain another basis for $N_{\eta(j)+1}$. Repeating this process for $1 \leqq j \leqq p$, and making other appropriate replacements, we arrive at vectors $x_{j}^{i}$ such that.

$$
\begin{array}{cc}
x_{1}^{1}, \cdots, x_{1}^{n} \text { are a basis for } N(A) \\
B x_{j}^{i}=A x_{j}^{i+1}, & 1 \leqq i \leqq \eta(j) \\
B x_{j}^{\eta(j)}=0, & 1 \leqq j \leqq p .
\end{array}
$$

For convenience, it is assumed that

$$
x_{j}^{i}=0 \quad \text { if } i>\eta(j) .
$$

If $1 \leqq j \leqq p$, let $P_{j}$ be the subspace of $X$ with basis $\left\{x_{j}^{1}, \cdots, x_{j}^{\eta(j)}\right.$. Let $Q_{j}$ be the subspace of $Y$ with basis $\left\{y_{j}^{1}, \cdots, y_{j}^{\eta(j)-1}\right\}$, where $y_{j}^{i}=$ $B x_{j}^{i}=A x_{j}^{i+1}$. Let $X_{1}=P_{1} \oplus \cdots \oplus P_{p}$ and $Y_{1}=Q_{1} \oplus \cdots \oplus Q_{p}$. Then $X_{1}$ and $Y_{1}$ satisfy all the conclusions of the theorem. To conclude the proof, it suffices to produce complementary subspaces to $X_{1}$ and $Y_{1}$ which also form an invariant pair.

We will construct functionals

$$
\begin{array}{ll}
\left\{g_{j}^{i}: 1 \leqq i \leqq \eta(j),\right. & 1 \leqq j \leqq p\} \text { on } X \text { and } \\
\left\{f_{j}^{i}: 1 \leqq i \leqq \eta(j)-1,\right. & 1 \leqq j \leqq p\} \text { on } Y \text { such that }
\end{array}
$$

the $f_{j}^{i}$ are in the domain of $A^{*}$ and

$$
\begin{array}{rlrl}
g_{j}^{i+1} & =A^{*} f_{j}^{i}, & & 1 \leqq i \leqq \eta(j)-1 \\
g_{j}^{i} & =B^{*} f_{j}^{i}, & & 1 \leqq i \leqq \eta(j)-1 \\
f_{J}^{i}\left(y_{k}^{q}\right) & =\delta_{i q} \delta_{j k}, & & 1 \leqq j, k \leqq n \\
& & 1 \leqq q \leqq i
\end{array}
$$




$$
\begin{array}{ll}
g_{j}^{i}\left(x_{k}^{q}\right)=\delta_{\imath q} \delta_{j k}, & 1 \leqq j, k \leqq n \\
& 1 \leqq q \leqq i .
\end{array}
$$

Let $g_{j}^{\eta(j)}$ be any functional on $X$ which satisfies (8). The other $g_{j}^{i}$ will be chosen by induction.

Suppose that $f_{k}^{q}$ and $g_{k}^{q}$ are chosen, for $q>i \geqq 1$, to satisfy (5) through (8). By (8), $g_{k}^{i+1}$ is orthogonal to $N(A)$, and so $g_{k}^{i+1}$ is in the closure of $R\left(A^{*}\right)$. Since $R(A)$ is closed, $R\left(A^{*}\right)$ is closed, and there is an $f_{k}^{i} \in D\left(A^{*}\right)$ for which $A^{*} f_{k}^{i}=g_{k}^{i+1}$. Let $g_{k}^{i}=B^{*} f_{k}^{i}$. Then (5) and (6) hold by definition.

To verify (7), we have for $q \leqq i$,

$$
\begin{aligned}
f_{j}^{i}\left(y_{k}^{q}\right) & =f_{j}^{i}\left(A x_{k}^{q+1}\right) \\
& =\left(A^{*} f_{j}^{i}\right)\left(x_{k}^{q+1}\right) \\
& =g_{j}^{i+1}\left(x_{k}^{q+1}\right)=\delta_{i q} \delta_{j k} .
\end{aligned}
$$

(8) is an immediate consequence of (7).

$$
\text { Let } \begin{aligned}
X_{0} & =\cap\left\{N\left(g_{j}^{i}\right): 1 \leqq i \leqq \eta(j),\right. & & 1 \leqq j \leqq p\} \\
Y_{0} & =\cap\left\{N\left(f_{j}^{i}\right): 1 \leqq i \leqq \eta(j)-1,\right. & & 1 \leqq j \leqq p\}
\end{aligned}
$$

From (7) and (8), it is clear that $X_{0} \cap X_{1}=\{0\}$ and $Y_{0} \cap Y_{1}=\{0\}$. Since the codimension of $X_{0}$ in $X$ is no greater than the number of functionals $g_{j}^{i}$ defining it, and since this number is the dimension of $X_{1}$, we must have $X=X_{0} \oplus X_{1}$. Similarly, $Y=Y_{0} \oplus Y_{1}$.

Suppose $x \in D(A) \cap X_{0}$. Then $f_{j}^{i}(A x)=\left(A^{*} f_{j}^{i}\right)(x)=g_{j}^{i+1}(x)=0$, and so $A x \in Y_{0}$. Similarly, $B x \in Y_{0}$, and $\left(X_{0}, Y_{0}\right)$ is an invariant pair for both $A$ and $B$.

Since $\left(X_{0}, Y_{0}\right)$ and $\left(X_{1}, Y_{1}\right)$ are invariant pairs, $N_{k}(A: B) \cap X_{0}=$ $N_{k}\left(A_{0}: B_{0}\right)$. For $k$ sufficiently large, $X_{1} \subseteq N_{k}(A: B)$, and so

$$
\begin{aligned}
\operatorname{dim}\left\{N_{k+1}\left(A_{0}: B_{0}\right) / N_{k}\left(A_{0}: B_{0}\right)\right\} & =\operatorname{dim}\left\{N_{k+1}(A: B) / N_{k}(A: B)\right\} \\
& =\sigma \\
& =\alpha(A)-p \\
& =\alpha\left(A_{0}\right) .
\end{aligned}
$$

This can occur only if $\operatorname{dim} N_{k}\left(A_{0}: B_{0}\right)=k \alpha\left(A_{0}\right)$ for all integers $k$. Hence $\mu\left(A_{0}: B_{0}\right)=\infty$.

3. Let $(P, Q)$ be an invariant pair of finite dimensional subspaces for $A$ and $B . \quad(P, Q)$ is an irreducible invariant pair of type $\nu$ if there are bases $\left\{x_{i}\right\}_{i=1}^{n}$ for $P$ and $\left\{y_{i}\right\}_{i=1}^{n}$ for $Q$ such that $B x_{i}=y_{i}$, $A x_{1}=0$, and $A x_{i}=y_{i-1}, 2 \leqq i \leqq n$.

$(P, Q)$ is an irreducible invariant pair of type $\mu$ if there are bases $\left\{x_{i}\right\}_{i=1}^{n}$ for $P$ and $\left\{y_{i}\right\}_{i=1}^{n-1}$ for $Q$ such that 


$$
\begin{aligned}
A x_{1} & =0=B x_{n} \\
A x_{i+1} & =y_{i}=B x_{i}, \quad 1 \leqq i \leqq n-1 .
\end{aligned}
$$

$(P, Q)$ is an irreducible invariant pair of type $\mu^{*}$ if there are bases $\left\{x_{i}\right\}_{i=1}^{n-1}$ for $P$ and $\left\{y_{i}\right\}_{i=1}^{n}$ for $Q$ such that

$$
\begin{aligned}
& B x_{i}=y_{i}, \quad 1 \leqq i \leqq n-1 \\
& A x_{i}=y_{i+1}, \quad 1 \leqq i \leqq n-1 \text {. }
\end{aligned}
$$

$(P, Q)$ is an invariant pair of type $\nu$ if $P=P_{1} \oplus \cdots \oplus P_{k}$ and $Q=Q_{1} \oplus \cdots \oplus Q_{k}$, where $\left(P_{j}, Q_{j}\right)$ is an irreducible invariant pair of type $\nu, 1 \leqq j \leqq k$. Invariant pairs of type $\mu$ or type $\mu^{*}$ are defined similarly.

It is straightforward to verify that if $(P, Q)$ is an (irreducible) invariant pair of type $\mu(A: B)$ (resp. $\left.\mu^{*}(A: B)\right)$, then $(P, Q)$ is an (irreducible) invariant pair of type $\mu(A-\lambda B: B)\left(\right.$ resp. $\left.\mu^{*}(A-\lambda B: B)\right)$, for all complex numbers $\lambda$. If $(P, Q)$ is an invariant pair of type $\mu$, then $\nu(A|P, B| P)=\infty$ and $\mu\left((A \mid P)^{*},(B \mid P)^{*}\right)=\infty$. If $(P, Q)$ is of type $\mu^{*}$, then $\nu(A|P, B| P)=\infty$ and $\mu(A|P, B| P)=\infty$.

THEOREM 2. Suppose $A$ and $B$ satisfy the standing hypothesis. Then there exist decompositions

$$
\begin{aligned}
& X=X_{0} \oplus X_{1} \oplus X_{2} \\
& Y=Y_{0} \oplus Y_{1} \oplus Y_{2}
\end{aligned}
$$

Where $\left(X_{0}, Y_{0}\right)$ is an invariant pair, $\left(X_{1}, Y_{1}\right)$ is an invariant pair of type $\mu$, and $\left(X_{2}, Y_{2}\right)$ is an invariant pair of type $\nu$. If $A_{0}$ and $B_{0}$ are the restrictions of $A$ and $B$ respectively to $X_{0}$, then $\nu\left(A_{0}, B_{0}\right)=\infty$ and $\mu\left(A_{0}, B_{0}\right)=\infty$.

Proof. Theorem 2 follows from Theorem 1 and Kato's Theorem 4 [1], after it is noted that the latter theorem, although stated only for bounded operators $B$, is valid under the less restrictive assumption that $D\left(B^{*}\right) \supseteqq D\left(A^{*}\right)$.

THEOREM 3. In addition to the standing hypotheses, suppose that $A$ is a Fredholm operator. Then there exist decompositions

$$
\begin{aligned}
& X=X_{0} \oplus X_{1} \oplus X_{2} \oplus X_{3} \\
& Y=Y_{0} \oplus Y_{1} \oplus Y_{2} \oplus Y_{3},
\end{aligned}
$$

where each $\left(X_{i}, Y_{i}\right)$ is an invariant pair, $\left(X_{1}, Y_{1}\right)$ is of type $\mu$, $\left(X_{2}, Y_{2}\right)$ is of type $\nu$, and $\left(X_{3}, Y_{3}\right)$ is of type $\mu^{*}$. If $A_{0}$ and $B_{0}$ are the restrictions of $A$ and $B$ to $X_{0}$, then $\nu\left(A_{0}: B_{0}\right)=\infty, \mu\left(A_{0}: B_{0}\right)=\infty$, $\mu\left(A_{0}^{*}: B_{0}^{*}\right)=\infty$, and $\nu\left(A_{0}^{*}: B_{0}^{*}\right)=\infty$. 
If $X^{*}=X_{0}^{*} \oplus X_{1}^{*} \oplus X_{2}^{*} \oplus X_{3}^{*}$ and $Y^{*}=Y_{0}^{*} \oplus Y_{1}^{*} \oplus Y_{2}^{*} \oplus Y_{3}^{*}$ are the corresponding decompositions of the adjoint spaces, then $\left(Y_{1}^{*}, X_{1}^{*}\right)$ is an invariant pair of type $\mu_{*}\left(A^{*}: B^{*}\right),\left(Y_{2}^{*}, X_{2}^{*}\right)$ is an invariant pair of type $\nu\left(A^{*}: B^{*}\right)$, and $\left(Y_{3}^{*}, X_{3}^{*}\right)$ is an invariant pair of type $\mu\left(A^{*}: B^{*}\right)$.

Proof. In view of Theorem 2, we may assume that $\mu(A: B)=\infty$ and $\nu(A: B)=\infty$. Then $\nu\left(A^{*}: B^{*}\right)=\infty$, and we can proceed to decompose $X^{*}$ and $Y^{*}$, as in the proof of Theorem 1. The only difficulty encountered is to produce vectors $x_{j}^{i}$ to span $X_{3}$ which actually lie in $D(A)$. An induction argument similar to that used in Theorem 1 to produce the $f_{j}^{i}$ and $g_{j}^{i}$ can also be employed in this case.

4. Let $\Phi^{+}(A: B)$ be the set of complex numbers $\lambda$ such that $A-\lambda B$ is a closed operator from $D(A)$ to $Y$, and such that $R(A-\lambda B)$ is closed and $\alpha(A-\lambda B)<\infty$. $\Phi^{+}(A: B)$ is an open subset of the complex plane which, by assumption, contains the point $\lambda=0$.

For all $\lambda \in \Phi^{+}(A: B)$, Theorems 1 and 2 are applicable to the operators $A-\lambda B$ and $B$. Also, for $\lambda \in \Phi^{+}(A: B)$ we define

$$
\begin{aligned}
\sigma_{k}(\lambda) & =\sigma_{k}(A-\lambda B: B) \\
\pi_{k}(\lambda) & =\pi_{k}(A-\lambda B: B) \\
\rho_{k}(\lambda) & =\rho_{k}(A-\lambda B: B) \\
\sigma(\lambda) & =\sigma(A-\lambda B: B) .
\end{aligned}
$$

THEOREM 4. Let $A$ and $B$ satisfy the standing hypotheses. There exists a decomposition

$$
\begin{aligned}
& X=X_{0} \oplus X_{1} \\
& Y=Y_{0} \oplus Y_{1}
\end{aligned}
$$

such that $\left(X_{0}, Y_{0}\right)$ is an invariant pair, and $\left(X_{1}, Y_{1}\right)$ is an invariant pair of type $\mu(A-\lambda B: B)$ for all complex numbers $\lambda$. If $A_{0}$ and $B_{0}$ are the restrictions of $A$ and $B$ to $X_{0}$, then $\mu\left(A_{0}-\lambda B_{0}: B_{0}\right)=\infty$ for all $\lambda \in \Phi^{+}(A: B)$ satisfying $\nu(A-\lambda B: B)=\infty$.

Proof. The points $\lambda \in \Phi^{+}(A: B)$ for which $\nu(A-\lambda B: B)<\infty$ form a discrete subset of $\Phi^{+}(A: B)$, and so there is a $\lambda^{\prime} \in \Phi^{+}$such that $\nu\left(A-\lambda^{\prime} B: B\right)=\infty$. Let $X=X_{0} \oplus X_{1}$ be the decomposition of Theorem 1 with respect to $A-\lambda^{\prime} B$ and $B$. Then $\left(X_{1}, Y_{1}\right)$ is an invariant pair of type $\mu(A-\lambda B: B)$ for all complex numbers $\lambda$, as remarked earlier.

If $\lambda \in \Phi^{+}(A: B)$ and $\nu(A-\lambda B: B)=\infty$, then $X_{0}$ and $Y_{0}$ cannot be decomposed further as in Theorem 1 , for such a decomposition would violate the fact that $\mu\left(A_{0}-\lambda^{\prime} B_{0}: B\right)=\infty$. Hence $\nu(A-\lambda B: B)=$ 
$\infty$ implies $\mu\left(A_{0}-\lambda B_{0}: B_{0}\right)=\infty$.

Let $D$ be the subset of $\Phi^{+}(A: B)$ of complex numbers $\lambda$ for which $\nu(A-\lambda B: B)<\infty$. $D$ is a discrete subset of $\Phi^{+}(A: B)$ with no limit points in $\Phi^{+}(A: B)$ (cf [1]).

THEOREM 5. $\mu(A-\lambda B: B)$ is a constant, either finite or infinite, for $\lambda \in \Phi^{+}(A: B)-D$.

Proof. In view of Theorem 4, it suffices to prove the theorem when $A$ and $B$ are operators in an invariant pair of type $\mu$. For this, it suffices to look at an irreducible invariant pair of type $\mu$. This case is easy to verify.

THEOREM 6. $\sigma(\lambda)$ is constant on each component of $\Phi^{+}(A: B)$.

Proof. It suffices to show that $\sigma(\lambda)$ is constant in a neighborhood of an arbitrary point $\lambda^{\prime} \in \Phi^{+}(A: B)$. Let $X=X_{0} \oplus X_{1} \oplus X_{2}$ and $Y=$ $Y_{0} \oplus Y_{1} \oplus Y_{2}$ be the decomposition of Theorem 2 with respect to $A-\lambda^{\prime} B$ and $B$. Then $\nu\left(A_{0}-\lambda B_{0}: B_{0}\right)=\infty$ for $\lambda$ near $\lambda^{\prime}$, and so $\sigma(\lambda)=$ $\alpha\left(A_{0}-\lambda B_{0}\right)$ for $\lambda$ near $\lambda^{\prime}$. By Theorem 3, [2], $\alpha\left(A_{0}-\lambda B_{0}\right)=$ $\alpha\left(A_{0}-\lambda^{\prime} B_{0}\right)$ for $\lambda$ near $\lambda^{\prime}$.

5. Let $X=X_{0} \oplus X_{1} \oplus X_{2}$ and $Y=Y_{0} \oplus Y_{1} \oplus Y_{2}$ be the decompositions of Theorem 2 with respect to $A$ and $B$. Let $\pi_{k}=\pi_{k}^{0}+\pi_{k}^{1}+\pi_{k}^{2}$ and $\rho_{k}=\rho_{k}^{0}+\rho_{k}^{1}+\rho_{k}^{2}$ be the corresponding decompositions of $\pi_{k}$ and $\rho_{k}$. Assume that $r$ is chosen small that $0<|\lambda|<r$ implies $\lambda \in \Phi^{+}(A: B)$ and $\nu(A-\lambda B: B)=\infty$. Then $\pi_{k}^{0}(\lambda)=k \sigma(\lambda)$ for $|\lambda|<r$. If $k$ is sufficiently large,

$$
\begin{aligned}
& \pi_{k}^{1}(\lambda)=\operatorname{dim} X_{1}, \quad|\lambda|<r \\
& \pi_{k}^{2}(\lambda)=\left\{\begin{aligned}
\operatorname{dim} X_{2}, & \lambda=0 \\
0, & 0<|\lambda|<r .
\end{aligned}\right.
\end{aligned}
$$

Also, $\rho_{k}^{0}(\lambda)=k \sigma(\lambda)$ for $|\lambda|<r$. For $k$ sufficiently large,

$$
\begin{aligned}
& \rho_{k}^{1}(\lambda)=\operatorname{dim} Y_{1} \\
& \rho_{k}^{2}(\lambda)=\left\{\begin{array}{cl}
\operatorname{dim} Y_{2}, & \lambda=0 \\
0, & 0<|\lambda|<r .
\end{array}\right.
\end{aligned}
$$

We define, for any $\lambda \in \Phi^{+}(A: B)$,

$$
\begin{aligned}
& \pi(\lambda)=\lim _{k \rightarrow \infty}\left[\pi_{k}(\lambda)-k \sigma(\lambda)\right] \\
& \rho(\lambda)=\lim _{k=\infty}\left[\rho_{k}(\lambda)-k \sigma(\lambda)\right]
\end{aligned}
$$

$\pi(\lambda)$ and $\rho(\lambda)$ correspond to $\tau(\lambda)$ defined in [1]. From the preced- 
ing, we deduce that

$$
\begin{aligned}
& \pi(\lambda)= \begin{cases}\operatorname{dim} X_{1}, & 0<|\lambda|<r \\
\operatorname{dim}\left(X_{1} \oplus X_{2}\right), & \lambda=0\end{cases} \\
& \rho(\lambda)= \begin{cases}\operatorname{dim} Y_{1}, & 0<|\lambda|<r \\
\operatorname{dim}\left(Y_{1} \oplus Y_{2}\right), & \lambda=0 .\end{cases}
\end{aligned}
$$

From these formulae, it follows that

$$
\alpha(A-\lambda B)=\sigma(\lambda)+\pi(\lambda)-\rho(\lambda), \quad 0<|\lambda|<r,
$$

for both sides of this expression are equal to

$$
\alpha\left(A_{0}-\lambda B_{0}\right)+\operatorname{dim} X_{1}-\operatorname{dim} Y_{1} .
$$

We will assume in the remainder of the discussion that $A$ is a Fredholm operator. The set of complex numbers $\lambda$ such that $A-\lambda B$ is a Fredholm operator will be denoted by $\Phi(A: B) . \quad \Phi(A: B)$ is an open subset of the complex plane, and consists of the union of those components of $\Phi^{+}(A: B)$ for which $R(A-\lambda B)$ is of finite codimension in $Y$, i.e., for which $\alpha\left(A^{*}-\lambda B^{*}\right)<\infty$.

The quantities $\pi_{k}^{*}(\lambda)=\pi_{k}\left(A^{*}-\lambda B^{*}: B^{*}\right), \rho_{k}^{*}(\lambda), \sigma^{*}(\lambda), \pi^{*}(\lambda)$ and $\rho^{*}(\lambda)$ are then well-defined for $\lambda \in \Phi(A: B)$. The formula for the adjoint operators corresponding to (5) is

$$
\alpha\left(A^{*}-\lambda B^{*}\right)=\sigma^{*}(\lambda)+\pi^{*}(\lambda)-\rho^{*}(\lambda), \quad 0<|\lambda|<r .
$$

Since $\alpha\left(A^{*}-\lambda B^{*}\right)=\beta(A-\lambda B)$, we have

$$
\begin{aligned}
& \kappa(A-\lambda B)=\left(\sigma(\lambda)-\sigma^{*}(\lambda)\right) \\
& \quad+\left(\pi(\lambda)-\pi^{*}(\lambda)\right)-\left(\rho(\lambda)-\rho^{*}(\lambda)\right) \quad 0<|\lambda|<r .
\end{aligned}
$$

In view of the decomposition of Theorem 3 , the jump discontinuity of $\pi^{*}$ at $\lambda=0$ is equal to that of $\pi$ at $\lambda=0$, i.e., they are both equal to $\operatorname{dim} X_{2}=\operatorname{dim} Y_{2}$. Hence (7) holds also for $\lambda=0$, and we arrive at the following theorem.

Theorem 7. For all $\lambda \in \Phi(A: B)$,

$$
\kappa(A-\lambda B)=\left(\sigma(\lambda)-\sigma^{*}(\lambda)\right)+\left(\pi(\lambda)-\pi^{*}(\lambda)\right)-\left(\rho(\lambda)-\rho^{*}(\lambda)\right) .
$$

Analogous formulae can be written down if it is assumed, further, that $B$ is a Fredholm operator. If $M(B)=\{0\}$ and $R(B)$ is dense in $Y_{1}$ then $\rho(\lambda)=\rho^{*}(\lambda)=\pi(\lambda)=\pi^{*}(\lambda)=0$, and Theorem 7 reduces to

$$
\kappa(A-\lambda B)=\sigma(\lambda)-\sigma^{*}(\lambda), \quad \lambda \in \Phi(A: B) .
$$

This latter formula is due to Kaniel and Schechter [1], when $X=Y$ and $B$ is the identity operator. 


\section{BIBLIOGRAPHY}

1. Kaniel and Schechter, Spectral theory for Fredholm operators, Comm. on Pure and Applied Math., vol. 16, no. 4 (1963), 423-448.

2. Kato, Perturbation theory for nullity, deficiency and other quantities of linear operators, J. d'Analyse Math. VI (1958), 261-322. 


\section{PACIFIC JOURNAL OF MATHEMATICS}

\section{EDITORS}

H. Samelson

Stanford University

Stanford, California

R. M. Blumenthal

University of Washington

Seattle, Washington 98105
J. Dugundu

University of Southern California Los Angeles, California 90007

*Richard Arens

University of California

Los Angeles, California 90024

\section{ASSOCIATE EDITORS}
E. F. BECKENBACH
B. H. NeumanN
F. WOLF
K. YOSIDA

\section{SUPPORTING INSTITUTIONS}

UNIVERSITY OF BRITISH COLUMBIA CALIFORNIA INSTITUTE OF TECHNOLOGY UNIVERSITY OF CALIFORNIA MONTANA STATE UNIVERSITY

UNIVERSITY OF NEVADA

NEW MEXICO STATE UNIVERSITY

OREGON STATE UNIVERSITY

UNIVERSITY OF OREGON

OSAKA UNIVERSITY

UNIVERSITY OF SOUTHERN CALIFORNIA
STANFORD UNIVERSITY

UNIVERSITY OF TOKYO

UNIVERSITY OF UTAH

WASHINGTON STATE UNIVERSITY

UNIVERSITY OF WASHINGTON

AMERICAN MATHEMATICAL SOCIETY CALIFORNIA RESEARCH CORPORATION SPACE TECHNOLOGY LABORATORIES NAVAL ORDNANCE TEST STATION 


\section{Pacific Journal of Mathematics}

\section{Vol. 15, No. $1 \quad$ September, 1965}

Donald Charles Benson, Unimodular solutions of infinite systems of linear

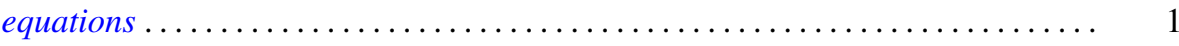

Richard Earl Block, Transitive groups of collineations on certain designs . . . . . . 13

Barry William Boehm, Existence of best rational Tchebycheff approximations .... . 19

Joseph Patrick Brannen, A note on Hausdorff's summation methods . . . . . . . . . . 29

Dennison Robert Brown, Topological semilattices on the two-cell ............ 35

Peter Southcott Bullen, Some inequalities for symmetric means . . . . . . . . . . 47

David Geoffrey Cantor, On arithmetic properties of coefficients of rational

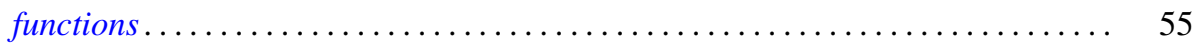

Luther Elic Claborn, Dedekind domains and rings of quotients . . . . . . . . . 59

Allan Clark, Homotopy commutativity and the Moore spectral sequence ........ 65

Allen Devinatz, The asymptotic nature of the solutions of certain linear systems of

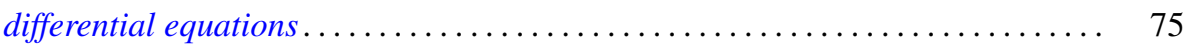

Robert E. Edwards, Approximation by convolutions ................... 85

Theodore William Gamelin, Decomposition theorems for Fredholm operators . . . . . 97

Edmond E. Granirer, On the invariant mean on topological semigroups and on

topological groups .................................. 107

Noel Justin Hicks, Closed vector fields . . . . . . . . . . . . . . . . . . . 141

Charles Ray Hobby and Ronald Pyke, Doubly stochastic operators obtained from

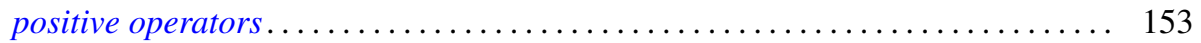

Robert Franklin Jolly, Concerning periodic subadditive functions . . . . . . . . . 159

Tosio Kato, Wave operators and unitary equivalence . . . . . . . . . . . . . . 171

Paul Katz and Ernst Gabor Straus, Infinite sums in algebraic structures . . . . . . . 181

Herbert Frederick Kreimer, Jr., On an extension of the Picard-Vessiot theory ...... 191

Radha Govinda Laha and Eugene Lukacs, On a linear form whose distribution is

identical with that of a monomial ......................... 207

Donald A. Ludwig, Singularities of superpositions of distributions . . . . . . . . . 215

Albert W. Marshall and Ingram Olkin, Norms and inequalities for condition

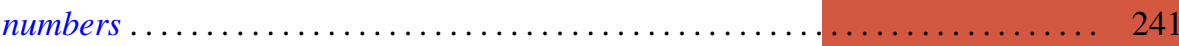

Horace Yomishi Mochizuki, Finitistic global dimension for rings . . . . . . . . . . 249

Robert Harvey Oehmke and Reuben Sandler, The collineation groups of division

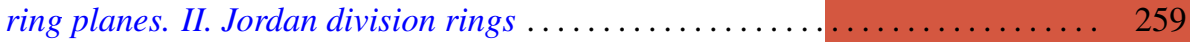

George H. Orland, On non-convex polyhedral surfaces in $E^{3} \ldots \ldots \ldots \ldots \ldots \ldots \ldots 267$

Theodore G. Ostrom, Collineation groups of semi-translation planes . . . . . . . . 273

Arthur Argyle Sagle, On anti-commutative algebras and general Lie triple

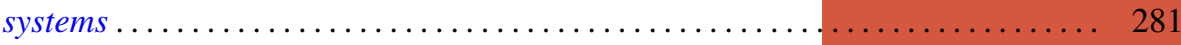

Laurent Siebenmann, A characterization of free projective planes . . . . . . . . . 293

Edward Silverman, Simple areas.................................. 299

James McLean Sloss, Chebyshev approximation to zero .................. 305

Robert S. Strichartz, Isometric isomorphisms of measure algebras . . . . . . . . . 315

Richard Joseph Turyn, Character sums and difference sets . . . . . . . . . . . . 319

L. E. Ward, Concerning Koch's theorem on the existence of arcs . . . . . . . . . . 347

Israel Zuckerman, A new measure of a partial differential field extension ......... 357 